\title{
Coherent Resonance of Saturated Absorption in Spectroscopy of Counterpropagating Waves
}

\author{
A. A. Chernenko ${ }^{1}$, E. G. Saprykin ${ }^{2}$, A. M. Shalagin ${ }^{2,3}$ \\ ${ }^{1}$ Institute of Semiconductor Physics, Siberian Branch, Russian Academy of Sciences, Novosibirsk, Russia \\ ${ }^{2}$ Institute of Automation and Electrometry, Siberian Branch, Russian Academy of Sciences, Novosibirsk, Russia \\ ${ }^{3}$ Novosibirsk State University, Novosibirsk, Russia \\ Email: chernen@isp.nsc.ru
}

Received 2013

\begin{abstract}
Results of theoretical researches of the saturated absorption resonance shape in a method of the probing field on V-type of transitions are represented. It is shown that in case of opposite circulary polarized optical fields the resonance is shown in the form of cross, and its form strongly depends on relaxation constants of levels and it can be represented as in the form of a dip, and absorption peak. Thus, the peak form has exclusively coherent character. Atomic transitions are offered, on which observation of the given effect is possible.
\end{abstract}

Keywords: Nonlinear Spectroscopy; Atomic Transition; Saturation Absorption; Cross Resonance; Light Wave

\section{Introduction}

It is known that action of the strong light fields on a resonant medium leads to nonlinear effects which are shown in the form of change of the density populations of resonant levels (effect of saturation), splitting of levels and an interference of levels - so-called nonlinear interferential effect (NIEF) as result of simultaneous absorption some photons [1]. The effect of saturation has exclusively incoherent character, and two others are caused by coherent processes. The method of a probing field [1,2] allows investigating the specified nonlinear effects. The resonance registered in this method in the absorption spectrum of the probing field by the resonant gas medium is shown in the form of a dip on the Doppler line contour and carries generally incoherent (populational) character. The coherent processes are shown in a form of the field broadening of the resonance spectrum (effect of level splitting) and as an additive in the dip amplitude (contribution of NIEF). And, the value of the NIEF contribution depends on the direction of propagation of the strong and probing light wave; effect is maximum at unidirectional waves, and in case of opposite directed waves the contribution of effect to resonance amplitude is strongly suppressed (in relation of the uniform width to the Doppler line width of atomic transition). So for the two-level medium under optimum conditions the share of NIEF in amplitude of a resonance doesn't exceed some percent [2]. In spite of the fact that researches on nonlinear spectroscopy of multilevel quantum systems are conducted already for a long time, a situation when the nonlinear resonance in opposite directed waves would be defined by only coherent processes, as far as we know, isn't found until now. There are cases, as in paper [3] where to the observed spectrum features of the nonlinear resonance, having the incoherent nature, NIEF manifestation was mistakenly attributed.

In this work we consider quantum system in which in case of the opposite directed light waves the nonlinear resonance having exclusively coherent nature (without a contribution of populated effects) with characteristic for coherent processes by an interferential form of a spectrum can be observed. Such situation is realized in the V-type of transitions the upper state of which has to breaks up only in the bottom state, and light waves have to be circular polarized with the counter direction of rotation of polarization vectors. A number of atomic transitions on which probably experimental observation of a resonance of coherent type is offered.

\section{Theoretical Model}

Let us consider the problem about the absorption spectrum of a probing field in a medium with V-type of transition in the presence of a strong field with the same frequency and opposite propagation direction. The scheme of transitions is presented in Figure 1. The strong wave is assumed to be plane (with the frequency $\omega$, wave vector $\boldsymbol{k}$, and electric field strength $\boldsymbol{E}$ ) and resonant to the atomic transition $m-n$ (with the transition frequency $\left.\omega_{m n}\right)$. Polarization of the strong wave is either linear or circular (separate case). The probe wave is also mono- 
chromatic (with the frequency $\omega_{\mu}=\omega$, the wave vector $\boldsymbol{k}_{\mu}$ $=-\boldsymbol{k}$, and the electric field strength $\mathbf{E}_{\mu}$ ) with circular polarization. When solving the problem, the saturation of a medium by the probing wave is taking into account under the assumption of its weakness as compared to the strong wave. The gas is assumed to be rarefied enough to neglect collisions. The medium is assumed to be optically thin.

We consider the problem in a coordinate system with the quantization exis along the direction of wave vector $\boldsymbol{k}$ of the strong field. In this coordinate system light fields induces transitions between magnetic sublevels (Figure 1) with variation $\Delta M= \pm 1$ (at linear polarization), or transitions with $\Delta M=+1$ or $\Delta M=-1$ (at circular polarization depending on a direction of its rotation).

In solving the problem we start from kinetic equations for a density matrix of the atomic system. According to [1], the dynamics of diagonal elements of the density matrix $\left(\rho_{i}=\rho_{i i}\right)$ in the model of relaxation constants is described by the system of equations:

$$
\begin{aligned}
\frac{\partial \rho_{i}}{\partial t}+\Gamma_{i} \rho_{i}= & Q_{i}+\sum_{k} \mathrm{~A}_{k i} \rho_{k}-2 \operatorname{Re}\left(i \sum_{j} V_{i j} \rho_{j i}\right) \\
& -2 \operatorname{Re}\left(i \sum_{j} V_{i j}^{\mu} \rho_{j i}\right)
\end{aligned}
$$

For off-diagonal elements of the density matrix the next system is valid:

$$
\frac{\partial \rho_{i k}}{\partial t}+\left(\Gamma_{i k}+i \omega_{i k}\right) \rho_{i k}=-i[V, \rho]_{i k}-i\left[V^{\mu}, \rho\right]_{i k}
$$

Here $\Gamma_{i}$ and $\Gamma_{i k}$ are the widths of the levels and transitions, $Q_{i}$ is the number of exitation acts of the $i$-th level per unit time, the summand $\sum_{k} \mathrm{~A}_{k i} \rho_{k}$ in system of equations (1) determines the spontaneous decay of the upper state $m$ to the lower state $n$ and is absent in the equations for the populations of upper levels, $\boldsymbol{V}$ and $\boldsymbol{V}^{\boldsymbol{\mu}}$ are operators of the interaction between the atom and the strong and probing fields. The operators are defined as $\boldsymbol{V}$ $=-\boldsymbol{G} \exp (i(\mathbf{k r}-\omega \mathrm{t}))+$ H.c. and $\boldsymbol{V}^{\boldsymbol{\mu}}=-\boldsymbol{G}^{\mu} \exp \left(i\left(\mathbf{k}_{\mu} \mathbf{r}-\omega_{\mu} \mathrm{t}\right)\right)$ + H.c., where the operators $\boldsymbol{G}=d E / \hbar$, and $\boldsymbol{G}^{\mu}=d E_{\mu} / \hbar$, and $d$ is the operator of the reduced dipole moment of atomic transition. Let's mark, that the given statement of the problem and the obtained solutions are fair both for transitions between exited states, and for a case, when the lower state $n$ is the ground state of atom. In this case the width of the lower state is determined by mean value of the interaction time of particles with the light fields.

Following [1], we seek solutions of the system of equations for the density matrix in the following form (the first harmonic approximation by the difference of wave frequencies $\left.\varepsilon=\omega_{\mu}-\omega\right)$ : the diagonal elements are found as $\rho_{i}=\rho_{i}^{0}+\rho_{i}^{+} \exp (i \varepsilon t)+\rho_{i}^{-} \exp (-i \varepsilon t)$; off-diagonal elements on the allowed and forbidden transitions ac- cordingly are found as $\rho_{i k}=R_{i k} \exp (-i \omega \mathrm{t})+R_{i k}{ }^{\mu} \exp \left(-i \omega_{\mu} \mathrm{t}\right)$ $+R_{i k}{ }^{s} \exp \left(-i \omega_{s} \mathrm{t}\right)$ and $\rho_{i k}={r_{i k}}{ }^{0}+r_{i k}{ }^{+} \exp (i \varepsilon \mathrm{t})+r_{i k}{ }^{-} \exp (-i \varepsilon \mathrm{t})$, where $\omega_{s}=2 \omega-\omega_{\mu}$. The substantiation of the given kind of solutions and their accuracies for the $V-, \Lambda$ - and $J=$ $1-J=1$ types of transitions are given by us in works $[4,5]$.

In the approximation of rotating optical fields the system of equations for the density matrix in the considered transition scheme in the stationary case is reduced to a system of algebraic equations for $\rho_{i}^{0}, \rho_{i}^{ \pm}, R_{i k}, R_{i k}{ }^{\mu}, R_{i k}{ }^{\mathrm{s}}$, $r_{i k}{ }^{0}$, and $r_{i k}{ }^{ \pm}$. Taking into account the hermiticity of these coefficients, we write only independent equations for a case of the linear polarized strong field. The equations for a case of the circular polarized strong field are easily received from reduced below, equating to zero one of the circular strength component of strong field.

In a case of the $\mathrm{V}$ - scheme (Figure 1) populations of the lower ( 0$)$ and the upper ( 1 and 2 ) levels are described by a following system of equations:

$$
\begin{gathered}
\Gamma_{n} \rho_{0}^{0}=Q_{0}+\sum_{k=1,2} A_{k 0} \rho_{k}^{0}+\sum_{k=1,2}\left(2 \operatorname{Re}\left(i G_{0 k} R_{k 0}\right)\right) \\
+2 \operatorname{Re}\left(i G_{02}^{\mu} R_{20}^{\mu}\right) \\
\left(\Gamma_{n}+i \varepsilon\right) \rho_{0}^{+}=\sum_{k=1,2} A_{k 0} \rho_{k}^{+}+i\left(G_{01} R_{10}^{s}+G_{02} R_{20}^{s}\right) \\
+i\left(G_{02}^{\mu} R_{20}-G_{20} R_{02}^{\mu}-G_{10} R_{01}^{\mu}\right) \\
\Gamma_{m} \rho_{1}^{0}=Q_{1}+2 \operatorname{Re}\left(i G_{10} R_{01}\right) \\
\left(\Gamma_{m}+i \varepsilon\right) \rho_{1}^{+}=i\left(G_{10} R_{01}^{\mu}-G_{01} R_{10}^{s}\right) \\
\Gamma_{m} \rho_{2}^{0}=Q_{2}+2 \operatorname{Re}\left(i G_{20} R_{02}\right)+2 \operatorname{Re}\left(i G_{20}^{\mu} R_{02}^{\mu}\right) \\
\left(\Gamma_{m}+i \varepsilon\right) \rho_{2}^{+}=i\left(G_{20} R_{02}^{\mu}-G_{02}^{\mu} R_{20}-G_{02} R_{20}^{s}\right)
\end{gathered}
$$

The system of equations for polarizations at the allowed (1-0 and 2-0 ) and at the forbidden 2-1 transitions has the next form:

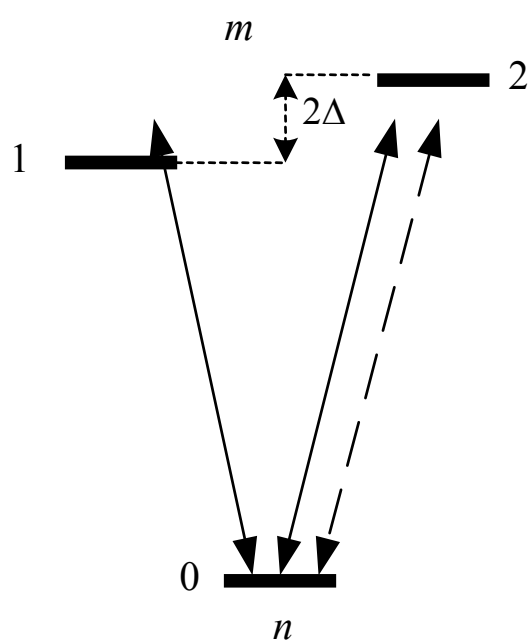

Figure 1. Scheme of interaction of light fields with V-type of atomic transitions, $\Delta$ - shift of levels. 


$$
\begin{gathered}
\left(\Gamma_{m n}+i \Omega_{10}\right) R_{01}=i G_{01}\left(\rho_{1}^{0}-\rho_{0}^{0}\right)+i G_{02} r_{21}^{0}+i G_{02}^{\mu} r_{21}^{-} \\
\left(\Gamma_{m n}+i\left(\Omega_{10}-\varepsilon\right)\right) R_{01}^{s}=i G_{01}\left(\rho_{1}^{-}-\rho_{0}^{-}\right)+i G_{02} r_{21}^{-} \\
\left(\Gamma_{m n}+i \Omega_{10}^{\mu}\right) R_{01}^{\mu}=i G_{10}\left(\rho_{1}^{+}-\rho_{0}^{+}\right)+i G_{02} r_{21}^{+}+i G_{02}^{\mu} r_{21}^{0} \\
\left(\Gamma_{m n}-i \Omega_{20}\right) R_{20}=-i G_{20}\left(\rho_{2}^{0}-\rho_{0}^{0}\right)-i G_{20}^{\mu}\left(\rho_{2}^{+}-\rho_{0}^{+}\right) \\
\quad-i G_{10} r_{21}^{0} \\
\left(\Gamma_{m n}-i\left(\Omega_{20}-\varepsilon\right)\right) R_{20}^{s}=-i G_{20}\left(\rho_{2}^{+}-\rho_{0}^{+}\right)-i G_{10} r_{21}^{+} \\
\left(\Gamma_{m n}-i \Omega_{20}^{\mu}\right) R_{20}^{\mu}=-i G_{20}\left(\rho_{2}^{+}-\rho_{0}^{+}\right)-i G_{20}^{\mu}\left(\rho_{2}^{0}-\rho_{0}^{0}\right) \\
\quad-i G_{10} r_{21}^{-} \\
\left(\Gamma_{m}+i \omega_{21}\right) r_{21}^{0}=i\left(G_{20} R_{01}-G_{01} R_{20}+G_{20}^{\mu} R_{01}^{\mu}\right) \\
\left(\Gamma_{m}+i\left(\omega_{21}+\varepsilon\right)\right) r_{21}^{+}=i\left(G_{20} R_{01}^{\mu}-G_{01} R_{20}^{s}\right) \\
\left(\Gamma_{m}+i\left(\omega_{21}-\varepsilon\right)\right) r_{21}^{-}=i\left(G_{20}^{\mu} R_{01}-G_{01} R_{20}^{\mu}+G_{20} R_{01}^{s}\right)
\end{gathered}
$$

In equations (3) - (5) $\Gamma_{n}$ and $\Gamma_{m}$ are the widths of the lower and upper levels, $\Gamma_{m n}$ is the width of the transition line, $\Gamma_{2 l}$ is the width of the forbidden transition between magnetic sublevels of the upper state; $\Omega_{i k}=\omega-\omega_{i k}$ and $\Omega^{\mu}{ }_{i k}=\omega_{\mu}-\omega_{i k}$ are the values of frequency detuning of the strong and probing fields from the frequencies $\omega_{i k}$ of transitions between the magnetic sublevels of the $m$ and $n$ states.

The motion of atoms is taken into account by the following changes in the equations:

$\Omega_{i k} \rightarrow \Omega_{i k}-\boldsymbol{k} \boldsymbol{v}, \Omega^{\mu}{ }_{i k} \rightarrow \Omega^{\mu}{ }_{i k}-\boldsymbol{k}_{\mu} \boldsymbol{v}$, and $\varepsilon \rightarrow \varepsilon-\left(\boldsymbol{k}_{\mu}-\right.$ $\boldsymbol{k}) \boldsymbol{v}$, where $\mathbf{v}$ is the velocity of atom. The situation of counter propagation of waves with equal frequencies was then analyzed: $\omega=\omega_{\mu}$, and $\boldsymbol{k}_{\mu}=-\boldsymbol{k}$.

The shape of the absorption line of the probing field (per atom) was determined by the following relation: $\alpha / \alpha_{0}=-\Gamma_{m n}<\operatorname{Re}\left(i\left(R_{20}{ }^{\mu}\right)\right) / G^{\mu}>$, where the designation $<\ldots>$ means the averaging by the Maxwell velocity distribution of particles, and $\alpha_{0}=4 \pi \omega_{m n} d^{2} / c \hbar^{2} \Gamma_{m n}$ is the resonant absorption cross section. In calculations the probabilities $A_{k i}$ of the decay of magnetic sublevels by each of the spontaneous channels were set to be similar and equal to $\mathrm{A}_{m n} / 2$.

\section{Results of Numerical Solution of the Initial Equations}

Stationary system of equations (3) - (5) that were obtained above for elements of the density matrix were solved numerically upon varying the values of width of the upper $\Gamma_{m}$ and lower $\Gamma_{n}$ atom levels, the parameter of the radiation branching from the upper level $a 0(a 0=$ $\left.\mathrm{A}_{m n} / \Gamma_{m}\right)$ and the intensities of optical fields using the relaxation characteristics of the $1 \underline{s}_{2}-2 p_{8}$ transition of the neon atom $\left(\mathrm{A}_{m n}=1.88 \times 10^{7} \mathrm{c}^{-1}, \Gamma_{m}=5.5 \times 10^{7} \mathrm{c}^{-1}, \Gamma_{n}=\right.$ $\left.10^{5} \div 10^{6} \mathrm{c}^{-1}, \Gamma_{m n}=\left(\Gamma_{m}+\Gamma_{n}\right) / 2\right)$. The values of level widths varied from the aforesaid values to $\Gamma_{m}=\Gamma_{n}=\Gamma_{m n}$, at the same time, the transition width $\Gamma_{m n}$ remained constant and the value of branching parameter a0 varied within the range $0.34 \div 1$. The line width of the forbidden transition between the sublevels of the upper state relied to width of the upper level. The Doppler line width is taken to be equal $k v_{\mathrm{T}}=5.2 \times 10^{9} \mathrm{c}^{-1}$, and the variation range of particle velocities at integration was $\pm 3 \mathrm{kv}_{\mathrm{T}}$ with a step $\Delta k v_{\mathrm{T}}=\left(10^{-3} \div 10^{-4}\right) k v_{\mathrm{T}}$. The saturation parameters of the strong $\left(\kappa_{s}\right)$ and probing $\left(\kappa_{p}\right)$ fields were chosen in the form

$\kappa_{s}=2(d E / 2 \hbar)^{2} /\left(\Gamma_{m n} \Gamma_{n}\right)$ and $\kappa_{p}=2\left(d E_{\mu} / 2 \hbar\right)^{2} /\left(\Gamma_{m n} \Gamma_{n}\right)$,

where $E$ and $E_{\mu}$ are the strengths of the circular components of the strong and probing fields and $d$ is the reduced dipole moment of transition. The values of saturation parameters varied within the limits $\kappa_{s} \leq 50$ and $\kappa_{p} \leq$ $\kappa_{s}$. Below, results are presented for small value of the saturation parameter of the probing field $\kappa_{p}=10^{-3}$.

\subsection{Strong Field of Linear Polarization}

Results of numerical simulation of the absorption spectrum of the probing wave of circular polarization in the presence of a strong saturating field of linear polarization are presented in Figures 2 and 3. When the upper state is degenerated the shape of the nonlinear resonance is represented as a dip in the center of the Doppler contour of the absorption line. Its amplitude and width depend on the saturation parameters of optical fields, on width of transition level and on the branching parameter a0. With elimination of the degeneracy (splitting) of the upper levels one can observe a dip of the saturated absorption at the shifted transition frequency in the shape of the absorption line of the circular polarized probing wave (named as PR, the sign of its shift is defined by the sign of circular polarization) and a cross resonance (CR) at the unshifted frequency $\omega_{m n}$. Amplitude and sign of the cross resonance depend essentially on the relation between the level widths $\Gamma_{m}$ and $\Gamma_{n}$ and on the branching parameter a0, at the same time, the effect of these characteristics on the parameters of the parent (PR) resonance is expressed much weaker.

It is seen from Figure 2 that, depending on the value of the branching parameter $\mathrm{a} 0$, the cross resonance can manifest itself not only as a dip, but also as an absorption peak. The maximal value of the peak amplitude is reached for the closed transition (for parameter $a 0=1$ ) both when the values of the level widths are similar $\left(\Gamma_{m} \sim \Gamma_{n}\right)$ and when they are considerably different $\left(\Gamma_{m}>>\Gamma_{n}\right)$. Thus it appears, that at a similar values of the saturation parameters $\kappa_{s}$ of the strong field, the peak amplitude in case of similar values of level width is considerably larger than the peak amplitude in case of a strong difference between the widths (curves 1 and 5). 


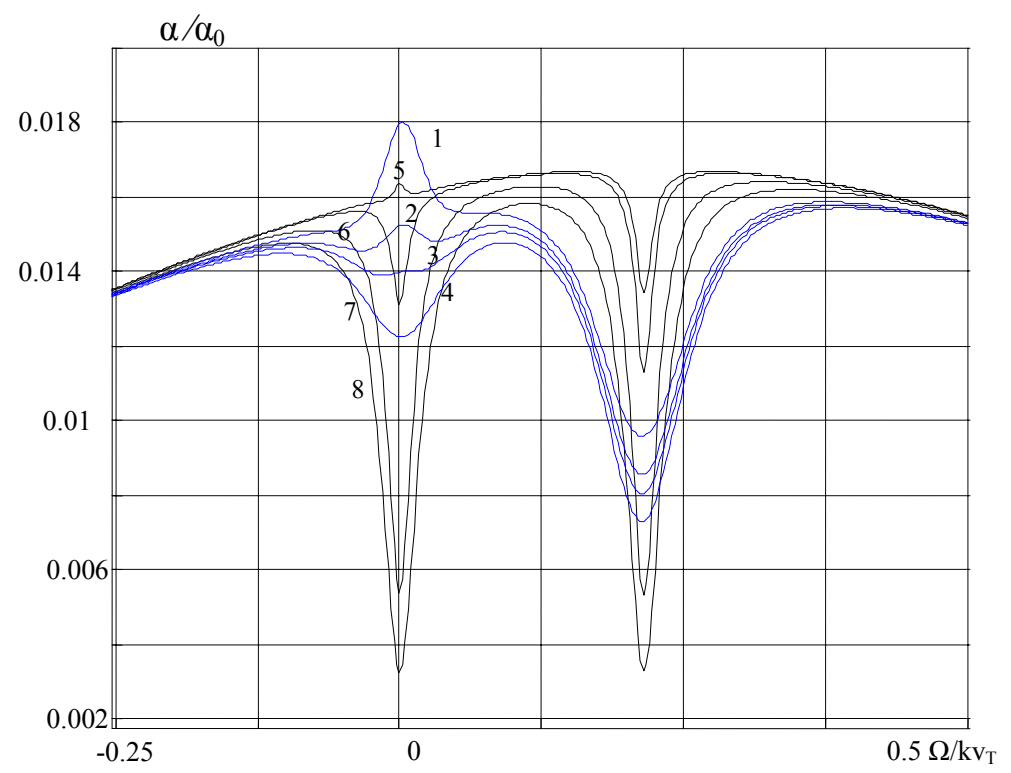

Figure 2. Resonance shapes at different values $a 0$ and $\Gamma_{n}: \Delta=40 \Gamma_{m n} ; \kappa_{s}=50, \kappa_{p}=10^{-3} ; \Gamma_{n}=\Gamma_{m n}(1-4), 0.04 \Gamma_{m n}(5-8) ; a 0=1(1$, 5), $0.98(6), 0.75(2,7), 0.6(3) ; 0.34(4,8)$.

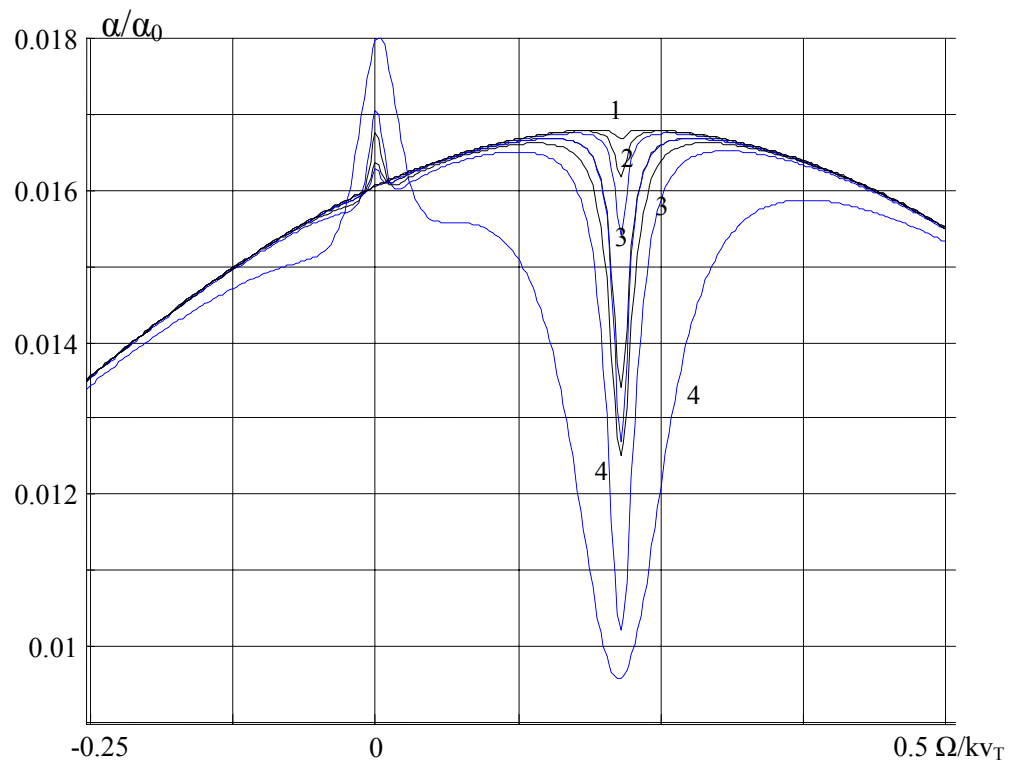

Figure 3. Behavior of the resonance shapes from intensity of the strong field: $\Delta=40 \Gamma_{m n} ; \mathrm{a} 0=1 ; \kappa_{p}=10^{-3}, \kappa_{s}=0.2(1) ; 1.0(2)$ 5.0 (3) 50 (4); $\Gamma_{n}=0.04 \Gamma_{m n}$ (continuous lines), $\Gamma_{m n}$ (dashed lines).

With a decrease in the value of the branching parameter cross resonance is transformed to a dip from the peak form. Moreover, in the case of similar values of level widths $\Gamma_{m}$ $=\Gamma_{n}$ the flip is observed for values of the branching parameter $a 0 \approx 0.6$; for the relation between the level widths $\Gamma_{m}>>\Gamma_{n}$ the change in the sign of the resonance amplitude occurs near the value $a 0 \sim 0.98$. Note also that, in case of similar values of the level widths the dip amplitude of the cross resonance is considerably less and the width is considerably larger than in case of when $\Gamma_{m}>>$ $\Gamma_{n}$ (compare curves 4 and 8).
Taking the approximation of a weak probing field and neglecting the polarization at the combination frequency $2 \omega-\omega_{\mu}$ one can obtain from the system of equations (3) (5) an analytical solution for polarization at the probing field frequency $R_{20}^{\mu}\left(\Omega_{\mu}\right)$ as:

$$
\begin{aligned}
& \left(\Gamma_{m n}-i \Omega_{20}^{\mu}+\frac{\left|G_{10}\right|^{2}}{\Gamma_{21}+i\left(\omega_{21}-\varepsilon\right)}\right) R_{20}^{\mu} \\
= & i G_{20}^{\mu}\left(\left(\rho_{0}^{0}-\rho_{2}^{0}\right)-\frac{\left(\rho_{0}^{0}-\rho_{1}^{0}\right)\left|G_{10}\right|^{2}}{\left(\Gamma_{m n}+i \Omega_{10}\right)\left(\Gamma_{21}+i\left(\omega_{21}-\varepsilon\right)\right)}\right)
\end{aligned}
$$


Here, the differences of level populations are determined via population of the lower level, branching parameter $a 0$ and rates of stimulated transitions $W_{i k}$ as:

$$
\begin{aligned}
& \rho_{0}^{0}-\rho_{2}^{0}=\rho_{0}^{0} /\left(1+W_{20} / \Gamma_{m}\right) ; \\
& \rho_{0}^{0}-\rho_{1}^{0}=\rho_{0}^{0} /\left(1+W_{10} / \Gamma_{m}\right) ; \\
& W_{i k} / \Gamma_{n}=\kappa_{s} \Gamma_{m n}^{2} /\left(\Gamma_{m n}^{2}+\Omega_{i k}^{2}\right) ; \\
& \rho_{0}^{0}=\frac{N_{0}}{1+\frac{(1-a 0) W_{10} / \Gamma_{n}}{1+W_{10} / \Gamma_{m}}+\frac{(1-a 0) W_{20} / \Gamma_{n}}{1+W_{20} / \Gamma_{m}}},
\end{aligned}
$$

where $N_{0}=Q_{0} / \Gamma_{n}$ is the population of the lower level in the absence of the strong field.

In the velocity (balanced) approximation the presence of a cross resonance is associated with amplitudes of the Bennett dips or peaks in the population of the common level. The specificity of the V-scheme is that the common level is the lower level and, in the absence of the spontaneous radiation from the upper level to the third levels (branching parameter $a 0=1$ ), a dip in the population of the common level is absent (it follows from (7) that $\rho_{0}^{0}=N_{0}$ ). It is compensated by the arrival of the spontaneously emitted particles. In this case the parent resonance is caused exclusively by the non-equilibrium population of the upper level. Under these conditions, it follows from solution (6) and averaging over the velocity distribution of particles that the populational part in the shape of the CR (the first summand in solution (6)) is represented by the Doppler contour and the peaks (Figure 2, curves 1 and 5) are caused only by coherent processes (the second summand in (6)), leading to an increase in the absorption coefficient. The performed calculations show that, in the given scheme of transitions, the contribution of coherent processes to the CR shape is maximal at equal values of level widths, and for case of $\Gamma_{m}>>\Gamma_{n}$ their contribution is less almost by an order of magnitude. This is illustrated in Figure 2 by the dependences for two relations between values of level widths both for the CR peak shape (curves 1 and 5) and for the dip (curves 4 and 8).

The effect of the saturating field intensity on the CR peak shape is shown in Figure 3. Here, for all relations between level widths and any values of the branching parameter, an increase in the strong field intensity leads to an increase in the CR amplitude and width both for the peak form and for the dip. However, the functional relation of amplitudes for the peak and for the dip form of the CR in the range of the used intensities of the saturating field appears different. If the CR amplitude in the form of a dip increases by a law close to the square root dependence, the relation of the amplitude of the coherent $\mathrm{CR}$ peak under these conditions is of linear character, with the square root dependence for the PR amplitude.

Let us mark the character of influence of the probing field intensity: an increase of the probing field intensity results in decreasing in the amplitude of the Doppler lining contour of the absorption line and in the amplitudes of the cross (for the dip and peak shapes) and parent resonances, thus an increase of the widths of these resonances is observed. However, here we did not take into account a capability of appearance of the spectrum features stipulated by spatial modulation of the nonlinear medium susceptibility in a field of standing waves. These problems were considered earlier (see, for example, activity [6,7] and reduced links in them). The nonlinear interference effects at the account of saturation by the probing field were calculated also in activity [8], but only for a case of unidirectional waves, in absence of the cross resonances.

\subsection{Strong Field of Circular Polarization}

The case of circular polarizations of the strong and probing wave is of interest. If the signs of rotation of electric field vectors of the strong and probing waves in a medium are similar, ${ }^{1}$ only the parent resonance will take place, its shift in the frequency scale will depend on the direction of rotation of field vectors and a value of splitting of the upper levels.

In case of the counter circular polarized vectors of fields only a cross resonance will be observed in the absorption line spectrum of the probing wave. The CR is located at the half sum of transition frequencies in the region of the interaction of counter propagating waves with the same group of moving atoms. The graphs corresponding to this case are presented in Figure 4. Here the saturated absorption line shapes, calculated for two values of splitting of the upper levels and a number of values of the atomic transition parameters are shown. It is visible, that for any value of level splitting within the limits of the Doppler line width the resonance is exhibited on the same light wave frequency. And, in the absence of branching parameter $(a 0=1)$ resonance is represented as the peak of absorption (curves 1 - 5). As it was marked above, in this case the population part of the $\mathrm{CR}$ is absent, and its shape is caused exclusively by the nonlinear coherent process (NIEF agrees with [1]). The presented relations (the most obvious are curves 2 and 4) demonstrate the alternating shape which is typical for the NIEF and a decrease in the effect with an increase in the difference between the level lifetimes. A decrease in the branching parameter a0 leads to appearance of the populational contribution and to transformation of the peak form into a dip (curves 5 - 8).

Thus, in case of the counter propagating and counter circular polarized waves the observed resonance of saturated

\footnotetext{
${ }^{1}$ In practice, these are experiments in which the probing wave is ob-
} tained as result of reflection from the mirror behind the cell with a gas. 


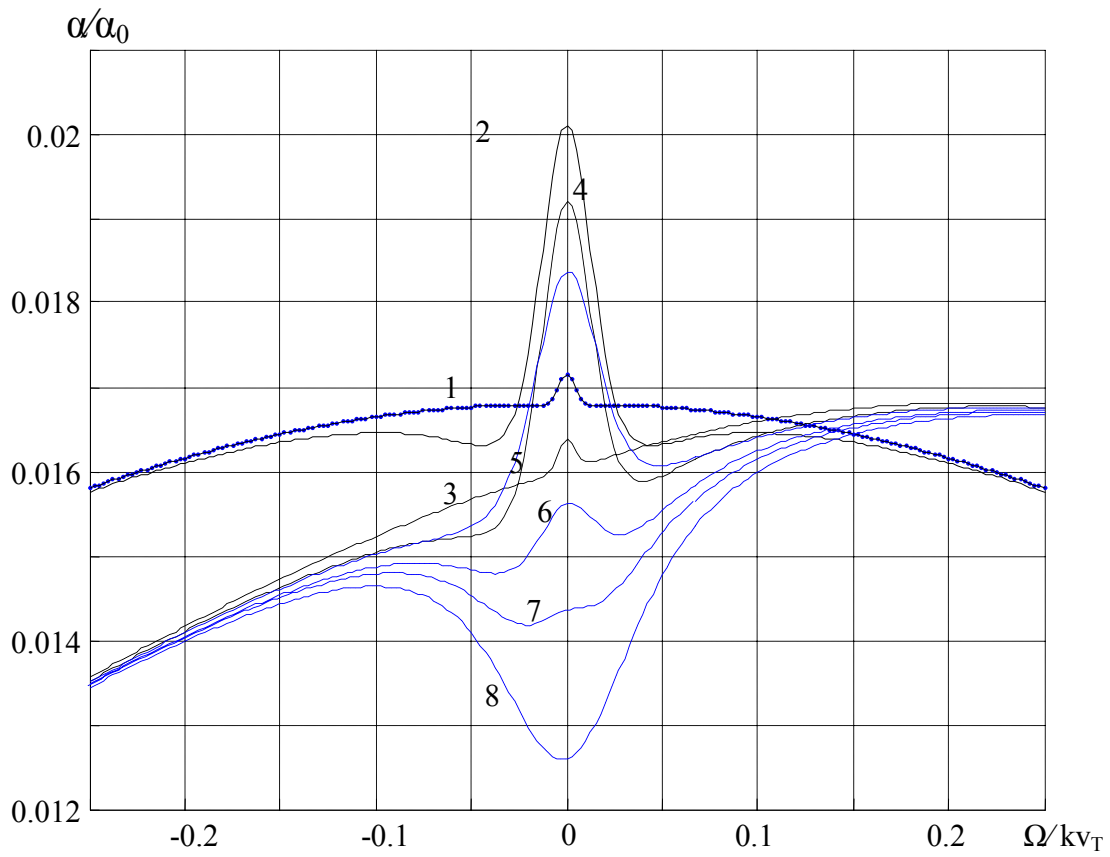

Figure 4. Shapes of resonances at orthogonally circular polarization of fields: $\kappa_{s}=50 ; \kappa_{p}=10^{-3} ; \Delta=0(1,2), \Delta=40 \Gamma_{m n}(3-8) ; a 0=1(1-4), 0.9(5)$, 0.75 (6), 0.6 (7), $0.34(8) ; \Gamma_{n}=\Gamma_{m n}(2,4-8), 0.04 \Gamma_{m n}(1,3)$.

absorption is a cross resonance actually. It is located in the center of the unshifted transition line at any value of splitting of the upper levels within the Doppler width of the line (in particular, in absence of splitting) and has under certain conditions extremely coherent nature. According to the results of [1], interference phenomena in the case of counter propagating waves that are absent in the approximation of first nonlinear corrections can appear in the next order with respect to saturation. This fact explains the aforementioned linear character of the increase of the coherent CR amplitude in Figure 2 with the square rooted dependence of the PR amplitude.

In conclusion we shall indicate on a capability of experimental observation of the coherent type saturated absorption resonance. The given resonance will be realized only in the V-type of transitions in atoms between states with the full moments $\mathrm{J}=0$ and $\mathrm{J}=1$, thus the upper state of transition should breaks up mainly on the lower state to supply the value of branching parameter a0 $\sim 1$. Transitions ${ }^{1} \mathrm{~S}_{0}-{ }^{1,3} \mathrm{P}_{1}$ from the ground state of the alkaline-earth metal atoms, and of the $\mathrm{Hg}, \mathrm{Cd}, \mathrm{Zn}$ and $\mathrm{Yb}$ atoms satisfy to these requirements. The wave lengths of these transitions are located in spectral area of generation of existing power tunable lasers (in the visible range), or their second harmonics (in the UV range).

\section{Acknowledgements}

This work was supported by the NSh-2979.2012.2 project and the Fundamental Optical Spectroscopy and Its Application Program (project 9.5) of the Branch of
Physical Sciences, Russian Academy of Sciences.

\section{REFERENCES}

[1] S. G. Rautian, G. I. Smirnov and A. M. Shalagin, "Nonlinear Resonances in Spectra of Atoms and Molecules," Nauka, Novosibirsk, 1979, [in Russian].

[2] V. S. Letokhov and V. P. Chebotayev, "Nonlinear Laser Spectroscopy of Ultrahigh Resolution," Nauka, Moscow, 1990. [in Russian].

[3] Yu. V. Bogdanova, "Nonlinear Resonances in the Signals of Magnetic and Frequency Scanning of Three-level $\Lambda$ and V-Systems," Optics of Atmosphere and Ocean, Vol 16, No. 7, 2003, pp. 567-575.

[4] E. G. Saprykin, A. A. Chernenko and A. M. Shalagin, "Effect of the Atomic Transition Parameters on Cross Resonance Shapes in Spectroscopy of Probing Field," Izvestiya Vuzov. Fizika, No. 2/2, 2011, pp. 255-268.

[5] E. G. Saprykin, A. A. Chernenko and A. M. Shalagin, "On the Shape of Cross Resonances in Counterpropagating Wave Spectroscopy," Optics and Spectroscopy, Vol 113, No. 5, 2012, pp. 585-597. doi:10.1134/S0030400X12080152

[6] M. G. Stepanov, "Autler-Townes Doublet Probed by Strong Field," Journal of Physycs B: Atomic, Molecular and Optical Physics, Vol. 32, 1999, pp. 649-661.

[7] S. A. Babin, E. V. Podivilov, V. V. Potapov, D. V. Churkin, and D. A. Shapiro," Nonlinear Resonance, Induced by Maximum Spatial Harmonics of Coherence," Journal of Experimental and Theoretical Physics, Vol. 121 , No. 4, 2002, pp. 807- 818 .

[8] A. K. Popov, S. A. Myslivets and Tomas, F. Geoges, "Nonlinear Interference Effects and All-Optical Switching to Optically Dense Inhomogeneously Brodened Media” Physical Review A, Vol. 71, 2005. 